Relations industrielles

Industrial Relations

\title{
La fonction ressources humaines en contexte québécois : perceptions et évolution
}

\section{Louis Gosselin}

Volume 50, numéro 1, 1995

URI : https://id.erudit.org/iderudit/050997ar

DOI : https://doi.org/10.7202/050997ar

Aller au sommaire du numéro

Éditeur(s)

Département des relations industrielles de l'Université Laval

ISSN

0034-379X (imprimé)

1703-8138 (numérique)

Découvrir la revue

Citer cet article

Gosselin, L. (1995). La fonction ressources humaines en contexte québécois : perceptions et évolution. Relations industrielles / Industrial Relations, 50(1), 186-209. https://doi.org/10.7202/050997ar
Résumé de l'article

Changements dans la législation du travail au Canada
Tous droits réservés (C) Département des relations industrielles de l'Universite Laval, 1995
Ce document est protégé par la loi sur le droit d'auteur. L'utilisation des services d'Érudit (y compris la reproduction) est assujettie à sa politique d'utilisation que vous pouvez consulter en ligne.

https://apropos.erudit.org/fr/usagers/politique-dutilisation/ 


\title{
La fonction ressources humaines en contexte québécois
}

\author{
Perceptions et évolution
}

\author{
LOUIS GOSSELIN
}

Dans un monde en changement, l'organisation s'ajuste aux besoins de son environnement. Dans le contexte des années 1980, la tangente prise par les entreprises privilégie la fonction ressources humaines en lui conférant un rôle prépondérant dans l'atteinte des objectifs organisationnels. Au cours de la dernière décennie, les chercheurs ont développé de nouvelles approches de gestion des ressources humaines et les praticiens ont vanté les mérites d'une préoccupation accrue de cette ressource dans l'organisation. Cependant, qu'en est-il réellement? Cette étude tente de retracer l'évolution de la fonction ressources humaines entre 1978 et 1989 au Québec et de vérifier si les perceptions qu'en ont les directeurs généraux et les directeurs des ressources humaines dans des entreprises du secteur secondaire privé ont sensiblement changé au cours de cette même période.

Quotidiennement confrontés à des changements majeurs dans leur environnement immédiat, les dirigeants d'entreprise doivent s'interroger sur les nouvelles règles du jeu qui leur sont imposées et s'ajuster à cette dynamique afin de maintenir l'entreprise comme maillon important dans le système social dans lequel elle évolue. Pour survivre, l'entreprise doit s'adapter à de nouveaux défis.

La permanence de l'incertitude dans la dynamique organisationnelle que doit gérer l'entreprise en fonction des objectifs à atteindre et des

- GosSelin, L., professeur, Département d'économie et de gestion, Université du Québec à Rimouski.

- Cette êtude a fait l'objet d'une thèse de doctorat présentée à l'Institut d'administration des entreprises, Université des sciences et techniques de Lille (France). 
ressources humaines disponibles, se traduit par des paradoxes fondamentaux (Louart 1990b; Martin 1990). Ces paradoxes sont définis par Louart comme "le fait que la réalité contienne des éléments ou des processus qui apparaissent contradictoires, sans doute parce qu'ils sont trop complexes à mentaliser pour une représentation humaine particulière " $(1990 \mathrm{~b}$ : 2). Ils font ressortir la difficulté de mettre en relation organisation et individu dans une société en changement sans saisir pleinement les interactions des personnes, ou pire encore, sans situer la compréhension qu'elles ont d'elles-mêmes.

C'est dans ce contexte que la fonction ressources humaines dans les organisations prend tout son sens. Elle permet l'adaptation de l'entreprise à son environnement, et cela sous plusieurs aspects. Vue et présentée désormais comme une fonction majeure à cause de l'accroissement de l'importance des ressources humaines dans le devenir organisationnel, elle doit voir accroître son pouvoir au même rythme que grandissent les responsabilités et les missions qui lui sont maintenant dévolues. Autant d'ordre environnemental qu'organisationnel, les forces et les tendances de l'avenir génèrent des attentes importantes à l'égard des ressources humajnes et requièrent l'aide et la participation de ces mêmes ressources pour permettre à l'entreprise de se développer.

\section{UNE VISION EVOLUTIVE DE LA FONCTION RESSOURCES HUMAINES}

Cette fonction à l'avant-scène de l'actualité organisationnelle n'est pas nouvelle, presque aussi ancienne que la révolution industrielle elle-même. Cependant, sa mission et son rôle ont changé. Avec le passage des décennies, un ensemble de facteurs organisationnels ont favorisé son évolution. Citons, entre autres, la dissociation du capital et du travail, la spécialisation des tâches (ou taylorisme), le mouvement des relations humaines et des sciences du comportement, et les relations du travail encadrées par la législation sociale des années de l'après-guerre.

Depuis le début du $\mathrm{XX}$ siècle, ces facteurs se sont succédé et nous ont conduits à l'histoire moderne de la gestion des ressources humaines que Wayne F. Cascio (1986: 44) présente en trois étapes : la phase tenue de dossiers (file maintenance stage), la phase exigences gouvernementales (government accountability stage), et la phase imputabilité organisationnelle (organizational accountability stage).

Au cours de la période qui se situe à mi-chemin des années 1960, la phase de tenue de dossiers met l'accent sur l'employé et le développement d'outils de gestion visant l'acquisition et le développement de la 
main-d'œuvre. Avec les lois touchant la discrimination, les régimes de pensions, la santé et la sécurité au travail, la seconde période qualifiée d'exigences gouvernementales couvre les années 1970 et fut surtout marquée par une gestion des règles et des conflits. L'intervention grandissante de l'État dans une société capitaliste incite les organisations à développer une gestion des ressources humaines plus conflictuelle. C'est la situation économique du début des années 1980 (taux d'intérêt élevé, inflation, récession, baisse de productivité, internationalisation des marchés) qui amorce le début de la troisième phase, celle dite de l'imputabilité organisationnelle, et qui incite les dirigeants à accorder une priorité à la productivité, à la qualité du produit et à la satisfaction de leurs ressources humaines. On doit alors situer la fonction ressources humaines dans un contexte organisationnel qui tient compte de son importance vitale: productivité et qualité de vie vont de pair.

Cette évolution de la fonction ressources humaines dans l'entreprise s'est également manifestée dans des changements de comportement, tels la participation à la prise de décision et aux comités d'entreprise, l'acceptation de responsabilités accrues, l'accès plus grand à l'information. Des changements importants se sont aussi produits dans les activités liées à la gestion des ressources humaines. Au fil du temps, les gestionnaires ont privilégié certaines activités, parfois au détriment de d'autres.

Mais dans leurs entreprises, quelles perceptions les directeurs des ressources humaines et les directeurs généraux ont-ils de cette fonction ressources humaines en pleine évolution? Où la situent-ils par rapport aux autres fonctions organisationnelles? Ces questions ont été posées en 1978 à des directeurs généraux et à des directeurs des ressources humaines d'entreprises québécoises de 200 employés et plus dans le secteur secondaire privé (voir Gosselin 1979). Cependant, si l'on tient compte de l'évolution rapide de cette fonction, qu'en est-il dix ans plus tard? Les résultats recueillis et le contenu des écrits publiés à cette époque permettaient de croire que cette fonction prendrait plus de place dans les organisations.

\section{LA QUESTION A L'ÉTUDE}

La fonction ressources humaines dans l'entreprise et les perceptions globales et particulières des directeurs généraux et des directeurs des ressources humaines de ces mêmes entreprises ont-elles évolué depuis la dernière décennie? Cette question globale comprend trois volets spécifiques : la notion d'évolution de la fonction ressources humaines elle-même, l'évolution des perceptions des acteurs interrogés et le rythme de transfert 
des connaissances entre la théorie et la pratique. Chaque volet fait l'objet d'une hypothèse :

Hypothèse I: la place, l'importance et les pratiques de la fonction ressources humaines dans les entreprises de plus de 200 employés du secteur secondaire ont évolué pendant la période 1978-1989 et l'équilibre entre les fonctions de l'entreprise en a été modifié.

Hypothèse II : en 1989, comme en 1978, dans les entreprises privées de plus de 200 employés du secteur secondaire, le directeur général entretient des perceptions de la fonction ressources humaines relativement similaires à celles du directeur des ressources humaines et ainsi les données utilisées présentent une vision juste de la réalité organisationnelle.

Hypothèse III: les activités de gestion relatives à la fonction ressources humaines passent lentement de la théorie à la pratique.

\section{LE MODËLE THEORIQUE}

En s'appuyant sur l'approche systémique, le modèle théorique qui en découle, tout en se voulant exploratoire, tente de concilier dans le temps et dans l'espace les perceptions des acteurs interrogés sur la fonction ressources humaines.

Le modèle (Figure 1) a la particularité de mettre en évidence la complexité de la fonction ressources humaines dans l'organisation autrement que par le service qui lui sert de support. Il utilise des variables d'aspect global pertinentes à cette fonction et des variables d'aspects particuliers liées aux activités de gestion qui lui sont propres pour donner, dans un premier temps, une image juste de la fonction observée et, en deuxième lieu, pour montrer son évolution dans le temps. Des variables modératrices, telles la taille de l'entreprise, son taux de syndicalisation et le budget attribué au service des ressources humaines, viendront particulariser cette évolution, qui devra aussi tenir compte des changements observés dans les profils des directeurs généraux et des directeurs des ressources humaines.

Une grille d'analyse a été établie en prenant comme point de départ des variables d'aspect global et d'aspects particuliers spécifiques à la fonction ressources humaines et elle a permis une lecture dans le temps (1978 et 1989) de la fonction étudiée. Ces variables ont été vérifiées suivant les perceptions des directeurs généraux et des directeurs des ressources humaines des entreprises de 200 employés et plus du secteur secondaire privé au Québec. L'évolution de la fonction ressources humaines peut alors être vérifiée et nuancée. 
FIGURE 1

Le modèle d'évolution de la fonction ressources humaines

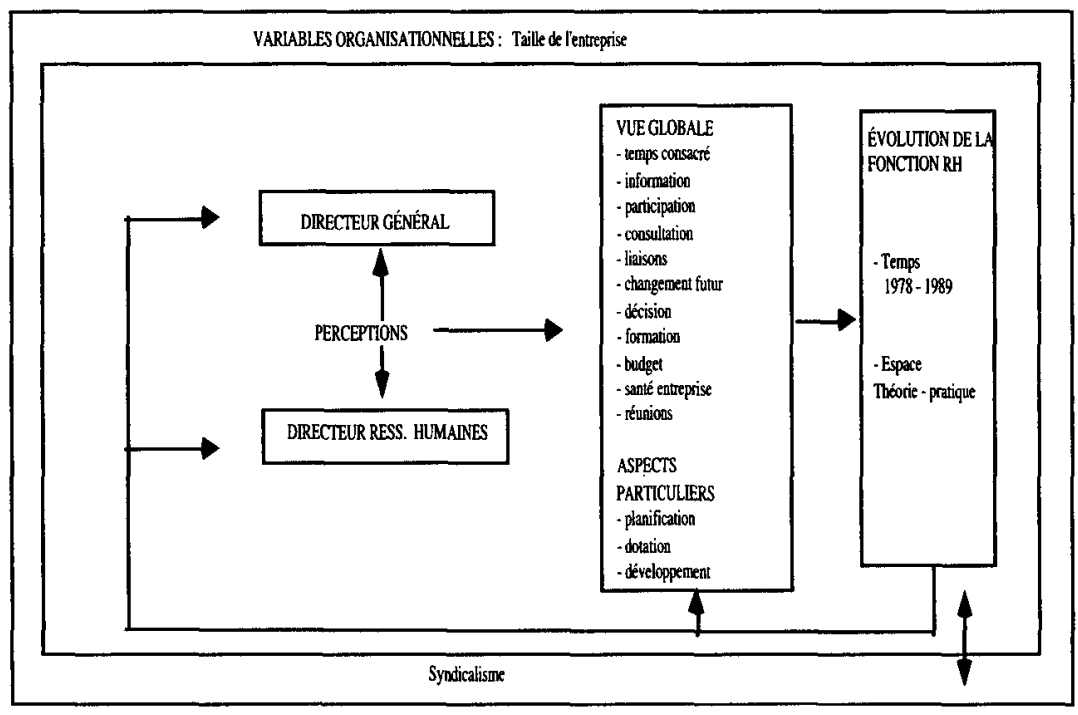

\section{La description du modele}

Le modèle proposé intègre les trois dimensions suivantes: les acteurs, le temps et l'espace.

\section{- L'intégration des acteurs}

Notre étude fait appel aux perceptions que des répondants se font de l'importance de la fonction ressources humaines dans l'entreprise. Il est fondamental d'obtenir les informations des hauts dirigeants de l'entreprise pour nous assurer d'une plus grande justesse de l'information. Ils ont normalement tendance à percevoir leur entreprise de façon globale contrairement aux cadres de niveau inférieur qui ont tendance à se faire de leur organisation une vision plus parcellaire, spécialisée et étroite.

Par contre, selon certains auteurs (Mintzberg 1984 ; St-Onge 1985), les perceptions des hauts dirigeants peuvent varier selon les qualifications de chacun. C'est pourquoi notre choix d'acteurs s'est porté sur le directeur général de l'entreprise et le directeur des ressources humaines, nous assurant ainsi d'une vision globale de la fonction par la direction générale et d'une vision plus spécialisée par le directeur des ressources humaines. Les acteurs choisis ont une grande connaissance de la fonction traitée et ils ont aussi une grande influence sur son devenir. 
Anne Tsui (1984) tente de démontrer dans son modèle $A$ Tripartite Framework of Personnel Department Effectiveness que pour avoir une bonne connaissance du service des ressources humaines d'une entreprise, il est nécessaire d'interroger tous les acteurs internes et externes en interaction avec ce service. Par contre, Wils et Labelle (1989), en faisant une application de ce mēme modèle, démontrent qu'il n'y a pas de différence significative sur le plan des perceptions du service des ressources humaines que se font les différents acteurs et qu'ainsi, il n'est pas nécessaire d'interroger l'ensemble des acteurs pour une meilleure compréhension de la fonction ressources humaines. Ceci justifie donc notre choix de n'interroger que le directeur général et le directeur des ressources humaines.

\section{-L'intégration dans le temps}

L'étude couvre une période de plus de dix ans, soit de 1978 à 1989 , et, compte tenu des changements socio-économiques et culturels qui ont marqué cette période, elle permet de vérifier les hypothèses énoncées. En effet, pendant ces dix ans on a assisté à une prise de conscience du rôle et de la place des ressources humaines dans l'organisation. De tels changements dans les valeurs des gens ont eu un impact sur les attitudes et les comportements des gestionnaires et des chercheurs.

\section{-L'intégration dans l'espace}

L'espace entre l'évolution théorique de la fonction ressources humaines et ses applications pratiques est signalé par un très grand nombre d'auteurs, tel Crozier (1989) qui, dans son ouvrage intitulé L'entreprise à l'écoute, conclut qu'il est préférable d'intervenir sur les moyens d'implantation plutôt que sur le développement de nouvelles théories en matière de gestion des ressources humaines. Le modèle développé permettra de vérifier ces affirmations en interrogeant les principaux intéressés dans l'organisation.

\section{LA MÉTHODOLOGIE}

Cette étude se situe au stade de la génération d'hypothèses; elle se veut inductive, plutôt descriptive que normative. Réalisé dans un contexte géographique et sociopolitique spécifique, le modèle théorique proposé ne peut se prêter à la généralisation mais contribue à fournir un cadre d'analyse susceptible d'être réutilisé dans des contextes différents. Si les hypothèses se confirment, il pourra alors servir de base à l'élaboration d'une théorie de l'évolution de la fonction ressources humaines dans les entreprises. Cette recherche comporte une limite importante, celle d'être 
tributaire d'un cadre méthodologique utilisé en 1978, qui, lui, demeure basé sur la réalité du temps.

\section{La population étudiée}

L'étude réalisée en 1978 sur l'état de la "fonction personnel " (appellation utilisée à l'époque) dans l'entreprise québécoise cherchait à délimiter la place qu'occupaient les ressources humaines dans les entreprises et à examiner comment les responsables de cette fonction étaient considérés. Au moment où les ressources humaines étaient gérées collectivement, nous voulions, à cette époque, connaître les préoccupations des dirigeants d'entreprise envers une gestion intégrée des ressources humaines dans leur organisation. Dix ans plus tard, après avoir refait une lecture de la réalité organisationnelle concernant la fonction ressources humaines, nous voulons établir une comparaison pour ces mêmes entreprises. Les entreprises retenues proviennent du secteur secondaire privé qui représente environ cinq pour cent des entreprises québécoises, mais qui embauchent le plus grand nombre de travailleurs. Elles se retrouvent surtout dans la grande région métropolitaine de Montréal, bien qu'elles soient présentes dans toutes les régions du Québec.

\section{L'échantillonnage}

Le directeur général et le directeur des ressources humaines constituent nos sources principales d'information puisque nous leur avons demandé de décrire les activités "ressources humaines " de leur entreprise pour chacun des aspects retenus. Comme en 1978, nous avons choisi, en 1989, les entreprises de 200 employés et plus comme objet d'observation. Nous considérons comme employés toute personne inscrite sur une liste de paye d'une entreprise, y compris les cadres.

Le secteur économique retenu est le secteur secondaire privé. Nous retrouvons là un groupe d'entreprises qui œuvrent dans toutes les activités de transformation, y incluant l'abattage et la transformation du bois. Les entreprises de notre échantillon sont dirigées par un conseil d'administration québécois (francophones et anglophones habitant au Québec) dans l'optique de faire ressortir davantage les particularités du Québec et d'exclure les entreprises étrangères gérées de l'extérieur sans relation autre qu'économique avec la société dans laquelle elles évoluent. Bien que cette particularité représentait une proportion plus significative en 1978 qu'en 1989, nous la maintenons afin de conserver à notre échantillon les mêmes caractéristiques.

Le nombre d'entreprises répondant aux caractéristiques mentionnées a augmenté entre 1978 et 1989 . De 145 entreprises du secteur secondaire privé ayant 200 employés et plus en 1978, on en compte 240 du même 
type en 1989. Cette augmentation de $65 \%$ par rapport à 1978 est accentuée par le fait que la taille des entreprises a aussi augmenté. Par contre, l'on remarque qu'il y a eu beaucoup de mouvements (fermeture d'entreprise, fusion, changement de raison sociale, etc.) parmi les entreprises identifiées en 1978, surtout parmi celles de petite taille.

\section{L'instrument de mesure}

En délimitant l'objet de notre étude et en explicitant les aspects retenus pour fins d'observation, nous avons utilisé le terme " variable " pour désigner les activités et les pratiques que l'on retrouve sous chacun de ces aspects (voir tableau 1). L'instrument que nous devons retenir doit chercher à rendre opérationnels ces variables et certains facteurs liés aux organisations et aux intervenants qui pourraient exercer une influence sur elles. Le questionnaire a été privilégié à l'entrevue et à l'observation participante à cause tout particulièrement de sa validité, de sa fidélité, de sa capacité à recueillir rapidement l'information et de son coût d'utilisation moins élevé.

TABLEAU 1

Les indicateurs de la fonction ressources humaines

\begin{tabular}{|c|c|c|c|}
\hline Vue globale & $\begin{array}{l}\text { Planification } \\
\text { des effectifs }\end{array}$ & $\begin{array}{l}\text { Recrutement, sélection, } \\
\text { placement }\end{array}$ & Formation \\
\hline $\begin{array}{l}\text { Temps consacré aux } \\
\text { activités de } \\
\text { planification } \\
\text { recrutement, formation } \\
\text { Diffusion d'information } \\
\text { Participation à des } \\
\text { comités dans le présent } \\
\text { et dans l'avenir } \\
\text { Consultation } \\
\text { Liaison avec les } \\
\text { autres fonctions } \\
\text { Pouvoir de décision } \\
\text { Évaluation des besoins } \\
\text { de formation des } \\
\text { responsables du service } \\
\text { du personnel } \\
\text { Budget consacré à cette } \\
\text { fonction } \\
\text { Critères de santé de } \\
\text { l'entreprise tels que } \\
\text { perçus par les dirigeants }\end{array}$ & $\begin{array}{l}\text { Existence d'une } \\
\text { politique } \\
\text { Durée de la } \\
\text { planification } \\
\text { Temps alloué à } \\
\text { cette activité } \\
\text { Participation } \\
\text { Pouvoir de décision } \\
\text { Liaison effective avec } \\
\text { d'autres activités et } \\
\text { d'autres fonctions }\end{array}$ & $\begin{array}{l}\text { Existence d'une telle } \\
\text { politique } \\
\text { Implication à la } \\
\text { préparation } \\
\text { Qualité de cette } \\
\text { politique } \\
\text { Temps consacré à } \\
\text { cette activité } \\
\text { Population visée par } \\
\text { cette politique } \\
\text { Décision concernant } \\
\text { cette activité }\end{array}$ & $\begin{array}{l}\text { Existence } \\
\text { Importance } \\
\text { Clientèle touchée } \\
\text { Type de formation } \\
\text { Période de temps } \\
\text { consacrée à cette } \\
\text { activité } \\
\text { Jugement sur la } \\
\text { nécessité de } \\
\text { cette activité } \\
\text { Pouvoir de } \\
\text { décision }\end{array}$ \\
\hline
\end{tabular}

Source : Gosselin (1979: 37). 
À titre de prétest, nous avons soumis le questionnaire à quelques directeurs généraux et à quelques directeurs des ressources humaines afin de déceler toutes les ambiguïtés qui auraient pu susciter des hésitations, des interrogations ou des commentaires défavorables. Afin de préserver la validité du questionnaire, aucune autre modification quant à la forme des questions, à l'ordre de présentation et à sa présentation globale n'a été apportée, bien que certains aspects du contenu pertinents en 1978 l'étaient moins en 1989.

\section{Le taux de réponses}

Le taux de réponses fut de $47,6 \%$ (122) en 1978 et de $44,5 \%$ (196) en 1989. Le taux de réponses des directeurs généraux et celui des directeurs des ressources humaines en 1978 et en 1989 sont présentés au tableau 2.

TABLEAU 2

Taux de réponses aux questionnaires

\begin{tabular}{ccccc}
\hline & \multicolumn{2}{c}{1978} & & \multicolumn{2}{c}{1989} \\
\cline { 4 - 5 } & D.G. & D.R.H. & D.G. & D.R.H. \\
\hline
\end{tabular}

Total des entreprises moins les inadmissibles (128 en 1978 et 220 en 1989) $61(47,6 \%) \quad 61(47,6 \%) \quad 76(34,5 \%) \quad 120(54,5 \%)$

Entreprises où les deux acteurs ont répondu $46(35,9 \%) \quad 46(35,9 \%) \quad 55(24,9 \%) \quad 55(24,9 \%)$

Il est aussi intéressant de signaler que dans respectivement $35,9 \%$ des cas en 1978 et $24,9 \%$ en 1989 , les répondants d'une même entreprise ont répondu au questionnaire et qu'avec ces résultats, il sera possible de vérifier l'évolution des perceptions des acteurs avec plus de certitude statistique (Tsui 1984).

\section{Les limites}

L'éventail des variables utilisées en vue d'analyser l'évolution de la fonction ressources humaines et celui des facteurs choisis pour en faire ressortir certaines particularités ne sont pas exhaustifs. Nous en avons privilégié un certain nombre de caractère managérial (aspect global) et 
fonctionnel (aspects particuliers) qui sont susceptibles de faire ressortir adéquatement les particularités de cette fonction.

Le choix du directeur général et du directeur des ressources humaines comme répondants se justifie bien théoriquement. Cependant, ce choix exclut d'autres acteurs tels les employés, les directeurs des autres services, les clients externes et les agents externes.

L'exclusion des entreprises de moins de 200 employés s'appuie sur plusieurs recherches (Mahé de Boislandelle 1988; Peretti 1989; Milkovich 1988 ; Gosselin 1979) qui démontrent que, bien que la fonction ressources humaines existe dans les petites entreprises, elle ne se retrouve pas dans un service spécifique et de ce fait, il n'existe pas de directeur des ressources humaines dans celles-ci.

De ce constat découle le choix du secteur d'activité privilégié et l'exclusion des secteurs primaire et tertiaire. En 1978, au Québec, le nombre d'entreprises de 200 employés et plus dans les secteurs primaire et tertiaire répondant aux caractéristiques de l'étude était insuffisant et les résultats obtenus ne pouvaient être significatifs. L'enquête de 1989 n'a pas retenu les entreprises des secteurs primaire et tertiaire exclues en 1978 afin de pouvoir comparer les résultats.

\section{LES RESULTATS}

Globalement, nous pouvons affirmer que la fonction ressources humaines au Québec a évolué très lentement au cours de la dernière décennie et qu'en 1989, les perceptions des directeurs généraux et celles des directeurs des ressources humaines étaient sensiblement les mêmes que celles constatées en 1978. Enfin, l'intégration des concepts théoriques propres à cette fonction ne s'est pas réalisée au rythme espéré tant par les chercheurs que par les gestionnaires. Les discours de ces derniers auraient laissé espérer un rythme d'intégration plus rapide des nouvelles approches en gestion des ressources humaines dans la pratique quotidienne; cependant la réalité est différente.

\section{Les organisations}

En 1978, comme en 1989, les informations ont été recueillies dans des entreprises représentatives de l'ensemble de la population étudiée. Selon leur taille et la répartition de leur capital-actions, les entreprises de l'étude reflètent la réalité québécoise. 
TABLEAU 3

Distribution en nombre et en pourcentage des directeurs généraux et des directeurs des ressources humaines selon la taille de l'entreprise (1978-1989)

\begin{tabular}{lccccc}
\hline & \multicolumn{2}{c}{ Directeurs généraux } & & \multicolumn{2}{c}{$\begin{array}{c}\text { Directeurs des } \\
\text { ressources humaines }\end{array}$} \\
\cline { 2 - 3 } \cline { 5 - 7 } Taille de l'entreprise & 1978 & 1989 & & 1978 & 1989 \\
\hline 200 à 499 & (40) $65,6 \%$ & (29) $38,2 \%$ & & (35) $57,4 \%$ & (55) $45,5 \%$ \\
500 à 999 & (11) $18,0 \%$ & (21) $27,6 \%$ & & (15) $24,6 \%$ & (29) $24,8 \%$ \\
1000 et plus & (10) $16,4 \%$ & (26) $34,2 \%$ & & (11) $18,0 \%$ & (36) $29,8 \%$ \\
\hline
\end{tabular}

Le coefficient de corrélation entre les entreprises contactées et les entreprises qui ont répondu à l'enquête se rapproche de l'unité (entre 0,81 et 0,996 ) et nous permet d'affirmer que, sur la base de la répartition des entreprises selon la taille, notre échantillon est représentatif de l'ensemble de la population étudiée.

Nous retrouvons des entreprises qui ont une taille plus grande en 1989 qu'en 1978. Le nombre de celles de plus de 500 employés a augmenté alors que le nombre d'entreprises de 200 à 499 employés a diminué. Ajoutons que les entreprises utilisent des capitaux québécois dans les mêmes proportions en 1978 et en 1989 et qu'elles sont fortement syndiquées ( $98 \%$ en 1978 et $83 \%$ en 1989) tout comme l'ensemble des entreprises du Québec de cette catégorie.

\section{Les acteurs}

Les résultats indiquent que nous sommes en présence d'un directeur général légèrement plus jeune, plus mobile et plus préoccupé par l'importance d'avoir près de lui un directeur des ressources humaines qui partage la même philosophie de gestion que la sienne. Quant au responsable des ressources humaines, il est aussi un peu plus jeune et mieux formé que ses prédécesseurs. Son champ de spécialisation est principalement en gestion et il est fortement orienté vers les ressources humaines.

Plus de la moitié des directeurs des ressources humaines en 1989 (56,3\%) détiennent un diplôme spécialisé de baccalauréat (16 ans de scolarité) comparativement à $30,5 \%$ en 1978 . Par sa formation, le directeur des ressources humaines a une vision multidisciplinaire de l'organisation et du travail et, sur le plan hiérarchique, il est plus près de la haute direction. 
FIGURE 2

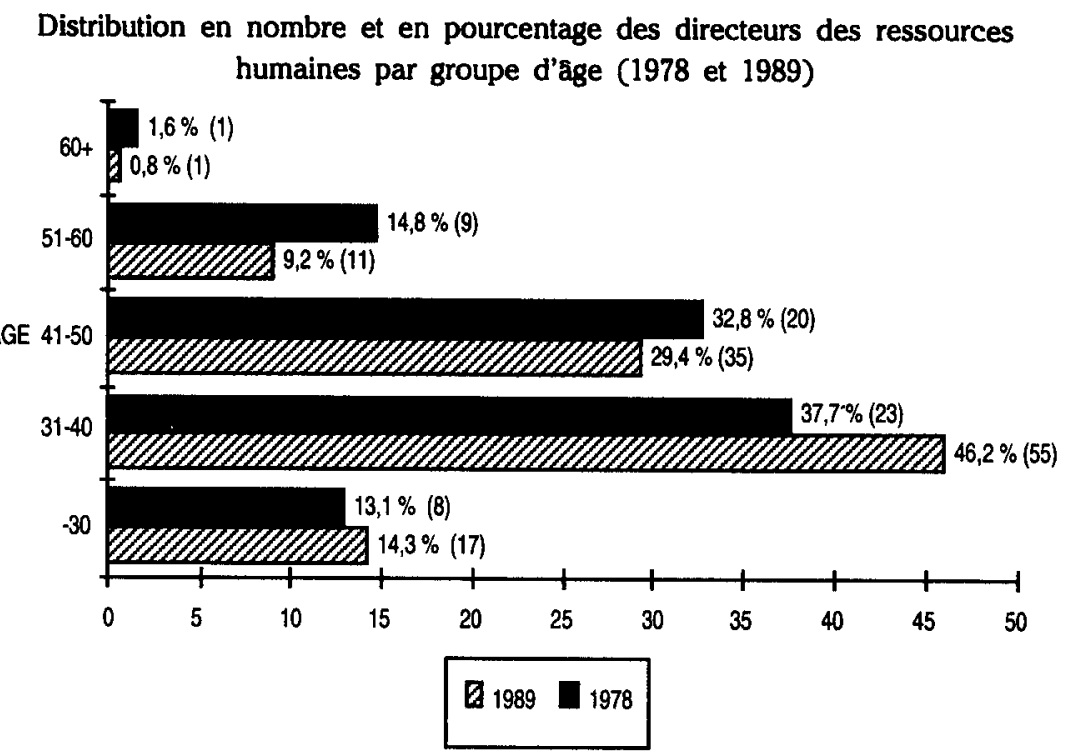

\section{L'évolution de la fonction ressources humaines}

Les profils des acteurs, modifiés par rapport à ceux de 1978, ont-ils eu un impact sur leur façon de gérer les ressources humaines au point de changer sensiblement la fonction ressources humaines? L'analyse qui précède laisse voir sous certains aspects une évolution de l'organisation et des acteurs interrogés au cours des dix dernières années au Québec. Déjà, à l'aide de variables personnelles et professionnelles liées aux acteurs, nous pouvons penser que les différences observées puissent avoir un impact sur la fonction ressources humaines elle-même.

\section{-L'aspect global}

L'aspect global de la fonction ressources humaines est représenté par un ensemble d'activités de gestion effectuées par les acteurs interrogés. Elles se répartissent en cinq rubriques : le partage du temps consacré aux activités ressources humaines; le pouvoir décisionnel des parties concernées; les liaisons interservices (communication); la participation des acteurs aux activités organisationnelles et le droit à l'information et à la consultation.

Sous chacun de ces thèmes se retrouve un ensemble d'activités spécifiques à la fonction ressources humaines relevant directement des responsables de cette même fonction. En relation les uns avec les autres, 
ces thèmes contribuent à présenter une image juste de la fonction étudiée.

Le partage du temps consacré aux activités ressources humaines. En 1989, les directeurs généraux et les directeurs des ressources humaines passent plus de temps à discuter ressources humaines et, contrairement à 1978, ils discutent davantage d'activités de gestion (planification des effectifs, formation de la main-d'œuvre) que d'activités de relations du travail, bien que celles-ci demeurent importantes.

Le pouvoir décisionnel des parties concernées. L'évolution du pouvoir décisionnel des acteurs pendant cette période a été très faible. Que ce soit à partir des perceptions du directeur général ou de celles du directeur des ressources humaines, l'exclusivité des décisions en matière de gestion des ressources humaines réservée au directeur général n'a pas changé de façon significative au cours des dix années ( $p>0.05)$.

Selon les directeurs des ressources humaines, $45 \%$ d'entre eux en 1978, comparativement à $41,5 \%$ en 1989, affirment que le directeur général se réserve l'exclusivité de certaines décisions dans le domaine de la gestion des ressources humaines. Les changements constatés entre 1978 et 1989 font surtout référence à une préoccupation accrue du directeur général pour les activités de dotation et de formation des ressources humaines au détriment des activités de relations du travail qu'il délaisse légèrement au profit du directeur des ressources humaines.

L'activité de négociation collective est celle qui fait davantage l'objet de décision exclusive du directeur général tant en 1978 qu'en 1989, bien que nous remarquons à cet égard une légère diminution (de 34,4\% à 31,6\%) pendant cette période. Nous constatons également que le directeur général se garde un pouvoir décisionnel exclusif en ce qui concerne la planification des effectifs. Comme c'est le cas pour la planification organisationnelle, la planification de la main-d'œeuvre est pour lui d'un plus grand intérêt que pour d'autres types d'activité, comme la gestion de la convention collective, qu'il confie davantage au directeur des ressources humaines.

Les liaisons interservices. La qualité des liaisons interservices jugée excellente en 1978 l'est toujours en 1989. Par contre, en 1978 comme en 1989, tant les directeurs des ressources humaines que les directeurs généraux souhaitent une amélioration de cette liaison. Les exigences accrues des gestionnaires envers la fonction ressources humaines pourraient expliquer en partie le désir d'améliorer les relations interservices.

La participation des acteurs aux activités organisationnelles. Bien que les deux acteurs interrogés participent dans une forte proportion, en 1978 comme en 1989, à des comités de gestion, ceux-ci sont différents en 1989. 
En effet, les directeurs des ressources humaines participent moins aux comités de relations du travail, de planification des opérations et d'orientations de l'organisation qu'en 1978.

FIGURE 3

Distribution en nombre et en pourcentage des directeurs des ressources humaines qui participent à l'un ou à l'autre des comités de gestion (1978 et 1989)

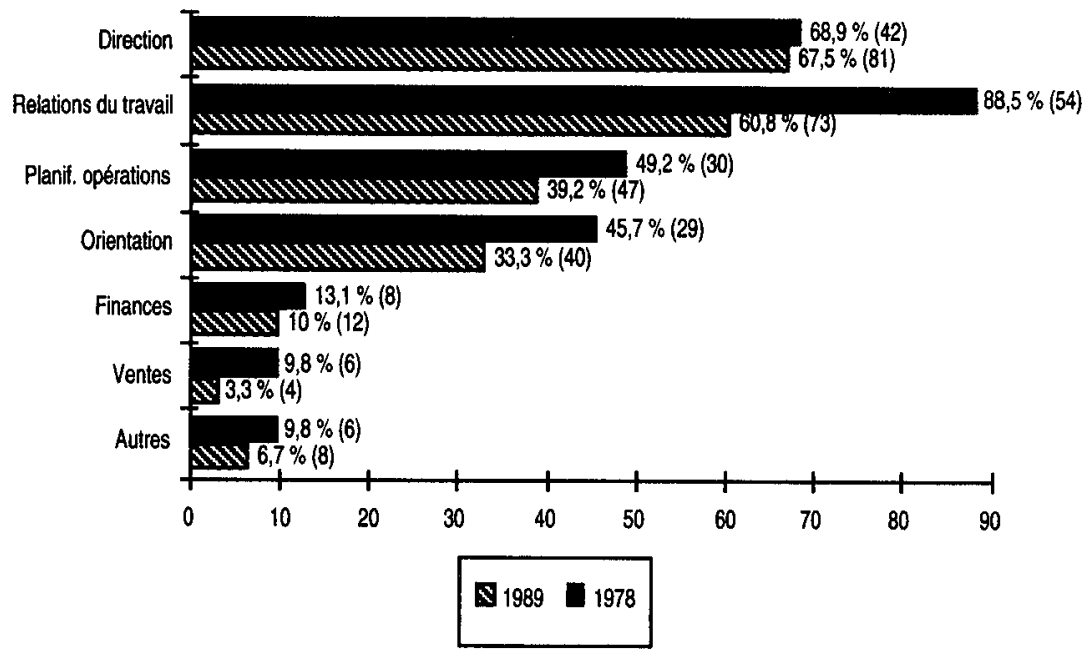

Cela laisse aussi supposer qu'ils délaisseraient, au profit de leurs subalternes, des responsabilités relatives aux relations du travail mais que, par contre, leur implication accrue au devenir organisationnel ne passerait pas par une plus grande participation à des comités décisionnels en cette matière.

Le droit à l'information et à la consultation. Une légère détérioration de la qualité de l'information, celle transmise par les directeurs des autres services au responsable des ressources humaines puis, par ce service, à la direction générale, est constatée en 1989. De plus, bien qu'il y ait une légère augmentation de la consultation portant sur les activités propres à la gestion des ressources humaines, nous remarquons une stagnation, voire une diminution, du niveau de consultation sur les activités d'intérêt général impliquant l'ensemble des fonctions de l'entreprise.

Sur l'ensemble des caractéristiques d'aspect global, l'évolution de la fonction ressources humaines est relativement faible. Cette fonction s'est 
développée dans ses activités, sa structure, sans pour autant s'intégrer davantage dans le devenir organisationnel. Certains résultats relatifs à l'analyse des activités spécifiques le démontrent.

\section{- Les aspects particuliers}

La planification des effectifs dans l'entreprise au cours des dix années étudiées n'a pas beaucoup évolué. Dans une grande proportion, mais de façon moins importante qu'en 1978, la haute direction et le responsable des ressources humaines effectuent en 1989 de la planification des effectifs à court et moyen termes. Ceux qui font de la planification des effectifs privilégient, autant en 1978 qu'en 1989, et dans une proportion dépassant $90 \%$, une planification à court et à moyen termes. Moins de $10 \%$ d'entre eux planifient à long terme, ce qui laisse supposer que très peu de dirigeants impliquent leur directeur des ressources humaines dans un processus de planification stratégique des ressources humaines. Cette constatation est confirmée par une étude réalisée en 1984 par Robert $\mathrm{H}$. Schwartz auprès de 203 membres affiliés à la Human Resource Planning Society. Par contre, en 1989, on confie davantage de pouvoir décisionnel en cette matière au directeur des ressources humaines et on admet que cette activité doit s'intégrer à des efforts de planification organisationnelle.

Par ailleurs, pour l'activité de dotation en ressources humaines, bien que peu de changements aient été constatés quant à l'existence ou non d'une politique écrite, de sa pertinence et de ses bénéficiaires, nous notons une implication accrue de tous les gestionnaires de l'entreprise dans son application quotidienne. Ceci apparaît clairement lorsqu'on considère la prise de décision en matière d'embauche. Ce type de décision qui revenait à $38,2 \%$ au directeur des ressources humaines n'est plus de son ressort exclusif en 1989 qu'à 25,9\%. Cette responsabilité qui incombe théoriquement au supérieur hiérarchique est assumée pratiquement par lui dans $65,7 \%$ des cas en 1989. L'analyse de cette variable démontre l'évolution du pouvoir décisionnel en matière de gestion des ressources humaines et plus spécifiquement en ce qui concerne les activités d'acquisition de la main-d'œuvre. Les gestionnaires de l'organisation prennent de plus en plus leurs responsabilités en cette matière marquant en cela une nette progression par rapport à 1978 .

Enfin, une légère évolution est aussi constatée lorsque nous analysons les activités de formation et de développement des ressources humaines. Un peu plus d'entreprises ont une politique de formation s'adressant à un plus grand nombre de personnes pour des durées pas nécessairement plus longues. On note une certaine régularité entre 1978 et 1989 quant aux raisons qui motivent les entreprises à faire de la formation et le rôle de 
conseiller dévolu au directeur des ressources humaines en 1978 est le même qu'en 1989 .

TABLEAU 4

Participation du directeur des ressources humaines aux différentes étapes du processus de formation (1978 et 1989)

\begin{tabular}{|c|c|c|c|c|c|c|}
\hline \multirow{2}{*}{$\begin{array}{l}\text { Étapes du processus } \\
\text { de formation }\end{array}$} & \multicolumn{2}{|c|}{ Décide } & \multicolumn{2}{|c|}{ Conseille } & \multicolumn{2}{|c|}{ Les deux } \\
\hline & 1978 & 1989 & 1978 & 1989 & 1978 & 1989 \\
\hline $\begin{array}{l}\text { Détermination des } \\
\text { besoins de formation }\end{array}$ & $\begin{array}{c}(12) \\
22,2 \%\end{array}$ & $\begin{array}{c}(25) \\
22,7 \%\end{array}$ & $\begin{array}{c}(41) \\
75,9 \%\end{array}$ & $\begin{array}{c}(83) \\
75,5 \%\end{array}$ & $\begin{array}{l}(1) \\
1,9 \%\end{array}$ & $\begin{array}{c}(2) \\
1,8 \%\end{array}$ \\
\hline Fixation des objectifs & $\begin{array}{c}(13) \\
24,5 \%\end{array}$ & $\begin{array}{c}(24) \\
22,2 \%\end{array}$ & $\begin{array}{c}(39) \\
73,6 \%\end{array}$ & $\begin{array}{c}(82) \\
75,9 \%\end{array}$ & $\begin{array}{c}(1) \\
1,9 \%\end{array}$ & $\begin{array}{c}(2) \\
1,9 \%\end{array}$ \\
\hline $\begin{array}{l}\text { Choix des individus } \\
\text { à former }\end{array}$ & $\begin{array}{c}(12) \\
21,4 \%\end{array}$ & $\begin{array}{c}(26) \\
23,9 \%\end{array}$ & $\begin{array}{c}(43) \\
76,8 \%\end{array}$ & $\begin{array}{c}(82) \\
75,2 \%\end{array}$ & $\begin{array}{l}(1) \\
1,8 \%\end{array}$ & $\begin{array}{c}(1) \\
0,9 \%\end{array}$ \\
\hline $\begin{array}{l}\text { Contenu de } \\
\text { programmes }\end{array}$ & $\begin{array}{c}(19) \\
34,5 \%\end{array}$ & $\begin{array}{c}(42) \\
38,5 \%\end{array}$ & $\begin{array}{c}(36) \\
65,5 \%\end{array}$ & $\begin{array}{c}(66) \\
60,6 \%\end{array}$ & - & $\begin{array}{c}(1) \\
0,9 \%\end{array}$ \\
\hline $\begin{array}{l}\text { Conduite des actions } \\
\text { de formation }\end{array}$ & $\begin{array}{c}(22) \\
41,5 \%\end{array}$ & $\begin{array}{c}(50) \\
46,7 \%\end{array}$ & $\begin{array}{c}(31) \\
58,5 \%\end{array}$ & $\begin{array}{c}(56) \\
52,3 \%\end{array}$ & - & $\begin{array}{c}(1) \\
0,9 \%\end{array}$ \\
\hline Choix des formateurs & $\begin{array}{c}(29) \\
52,7 \%\end{array}$ & $\begin{array}{c}(61) \\
57,0 \%\end{array}$ & $\begin{array}{c}(26) \\
47,3 \%\end{array}$ & $\begin{array}{c}(45) \\
42,1 \%\end{array}$ & - & $\begin{array}{c}(1) \\
0,9 \%\end{array}$ \\
\hline $\begin{array}{l}\text { Coûts impliqués dans } \\
\text { l'action de formation }\end{array}$ & $\begin{array}{l}(24) \\
44,4 \%\end{array}$ & $\begin{array}{l}(50) \\
47,2 \%\end{array}$ & $\begin{array}{c}(30) \\
55,6 \%\end{array}$ & $\begin{array}{c}(55) \\
51,9 \%\end{array}$ & - & $\begin{array}{c}(1) \\
0,9 \%\end{array}$ \\
\hline
\end{tabular}

Autant en 1978 qu'en 1989, la quasi unanimité des responsables des ressources humaines insistent sur l'importance de réaliser des activités de formation au profit des organisations. Ils sont aussi d'accord pour dire que la formation contribue à l'accroissement de la productivité et favorise la motivation du personnel.

\section{- Les effets des variables modératrices sur la fonction ressources humaines}

À quoi peut-on attribuer la légère évolution constatée de la fonction ressources humaines? Trois variables modératrices ont retenu notre attention: la taille de l'entreprise, son taux de syndicalisation et le pourcentage du budget attribué à la fonction ressources humaines. 
L'analyse des résultats, aussi bien en 1978 qu'en 1989, démontre que peu importe la taille de l'entreprise (200 à 499 employés et 500 employés et plus), les directeurs généraux et les directeurs des ressources humaines perçoivent de façon identique la fonction ressources humaines. Cette variable n'a donc aucun impact sur l'évolution de la fonction étudiée.

L'analyse de l'impact du taux de syndicalisation sur l'évolution de la fonction ressources humaines est plus complexe due au fait qu'une très grande proportion des entreprises étaient syndiquées et que la comparaison statistique s'avère impossible. Par contre, l'évolution de la fonction ressources humaines doit tenir compte de l'évolution des philosophies des centrales syndicales québécoises eu égard aux conflits. Au cours de la décennie à l'étude, ces dernières ont développé des approches participatives favorisant le maintien des emplois et le développement des organisations. Pris sous cet angle, le syndicalisme québécois contribue à sa façon au développement de la fonction ressources humaines.

Enfin, l'évolution de cette fonction n'est pas due à une augmentation du budget qui lui est consacré. En effet, pour la période étudiée, le pourcentage de budget alloué à cette fonction n'a pas augmenté de façon significative. Par contre, il est important de souligner que les entreprises qui consacrent plus de $5 \%$ de leur budget au service des ressources humaines privilégient les activités de planification des effectifs et de formation de la main-d'œuvre et confient plus de responsabilités au directeur des ressources humaines en cette matière. Malgré cette exception, le changement dans les comportements des gestionnaires, qui se traduit par une légère évolution de la fonction ressources humaines, doit se faire sans un support financier accru.

\section{Les perceptions}

Le modèle théorique et l'étude empirique cherchant à démontrer l'évolution de la fonction ressources humaines dans les entreprises québécoises ont comme pierre d'assise les perceptions des acteurs. C'est par la vision des directeurs généraux et des directeurs des ressources humaines que nous démontrons les hypothèses formulées au départ sur l'évolution de la fonction ressources humaines. D'où l'importance de vérifier si les perceptions des acteurs interrogés sont concordantes et si elles se sont modifiées dans le temps.

Le choix des acteurs permet d'avoir une lecture juste de la réalité observée (Tsui 1987; Wils et al. 1989). Quant aux nuances sur le plan des perceptions de la fonction ressources humaines entre les acteurs interrogés, nuances décrites comme peu importantes en 1978 (Gosselin 1979), elles ont peu changé en 1989. Lorsque nous comparons les réponses données 
par ces acteurs, autant en 1978 qu'en 1989, nous constatons dans l'ensemble très peu de différences de perceptions.

L'analyse des variables d'aspect global et celles d'aspects particuliers vues sous l'angle des perceptions des acteurs interrogés démontre, dans un premier temps, que les directeurs généraux et les directeurs des ressources humaines ont sensiblement la même vision de leur réalité organisationnelle en matière de gestion des ressources humaines. De plus, lorsque nous constatons certaines différences, elles sont maintenues dans le temps, c'està-dire que, en 1978 comme en 1989, les personnes interrogées maintiennent leur vision discordante au sujet de certaines variables spécifiques à certaines activités de gestion.

A titre d'exemple, lorsque nous analysons l'évolution des perceptions du directeur général entre 1978 et 1989 relativement à la participation du directeur des ressources humaines aux comités de gestion de l'entreprise, près de neuf directeurs généraux sur dix affirment que leur directeur des ressources humaines participe à ces comités. Cette vision de la réalité n'est confirmée que par $84,4 \%$ des directeurs des ressources humaines en 1978 et $76,4 \%$ en 1989 . Il y a une différence de perceptions minime entre les deux acteurs sur ce thème, et elle se maintient dans le temps.

Cette constatation vient expliquer le fait que les acteurs ont une vision qui colle à leur réalité et que leur désaccord, à certains égards, s'explique beaucoup plus par le poste qu'ils occupent dans l'organisation que par leur profil de gestionnaire. L'analyse vient confirmer que la description faite par les acteurs interrogés est représentative de la réalité organisationnelle. Les activités de gestion des ressources humaines décrites sont effectivement les activités existantes dans les entreprises et la qualité des relations perçue par un des acteurs interrogés est presque toujours confirmée par l'autre. Les différences observées quant aux réponses des directeurs généraux et des directeurs des ressources humaines d'une même entreprise sont si minimes qu'elles ne remettent aucunement en cause la qualité des données analysées. Au contraire, elles ne font que les confirmer et leur donnent plus de crédibilité.

Enfin, aussi bien en 1978 qu'en 1989, l'effet de la taille de l'entreprise, comme variable modératrice dans l'ensemble des variables étudiées, ne démontre aucune différence significative entre les perceptions des acteurs interrogés. La vision de ces derniers est constante et elle est représentative de la réalité observée.

\section{Le rythme d'intégration des concepts théoriques à la réalité organisationnelle}

De toutes les fonctions organisationnelles, la fonction ressources humaines est sans contredit la fonction de la décennie. On prend 
conscience que l'entreprise n'est pas seulement un ensemble de fonctions structurées mais un "construit humain" (LeBoterf 1988) qui a sa propre personnalité (culture) et qui fait face quotidiennement à une réalité changeante à laquelle elle doit s'adapter. Dans ce contexte, à quoi pouvons-nous attribuer la faible évolution de la fonction ressources humaines dans les entreprises québécoises du secteur secondaire privé? Bien sûr, toutes les entreprises ne sont pas innovatrices et, même si des expériences dans ce sens se poursuivent, on constate la présence d'un fort degré de conservatisme qui s'incruste avec le temps (Lemelin et Rondeau 1990). Tenant compte des nombreux développements dans le domaine de la gestion des ressources humaines sur une période de dix ans, auraitil été possible de s'attendre à une différence aussi importante entre la théorie et la pratique ?

Le management, décrit par Crozier dans L'entreprise à l'écoute: Apprendre le management post-industriel, comme "art de la pratique s'il en est, n'est pas l'application d'une doctrine mais la réponse donnée à des problèmes et à des contradictions réelles, en fonction des capacités humaines à concevoir et à mettre en œuvre des pratiques appropriées ". Il enchaîne en disant que sans pensée managériale nouvelle, " on ne peut animer une pratique originale "(Crozier 1989: 35). Cette réalité laisse croire que, si des formes organisationnelles nouvelles naissent, elles se traduiront par des pratiques cohérentes dans les organisations et contribueront au devenir de l'entreprise.

Les années 1980 ont été marquées par l'apparition " d'une multitude de techniques ou approches toutes faites, présentées comme innovantes et résolvantes " (Hermel 1990: 14) et, pourtant, la réalité observée laisse voir une fonction ressources humaines peu différente de celle de la décennie qui l'a précédée. Bien qu'une légère évolution soit constatée, ceux qui façonnent l'entreprise et la fonction ressources humaines perçoivent cette dernière, en 1989, comme une fonction légèrement plus importante qu'elle l'était en 1978. Les perceptions que les directeurs généraux et les directeurs des ressources humaines s'en font rejoignent difficilement le discours qu'ils tiennent à son égard.

Les dirigeants d'entreprise se laissent-ils prendre au phénomène de la mode? Prenant leur désir pour des réalités véhiculées dans leur environnement, plus que dans leur organisation, ils acceptent le changement sans pour autant l'initier. Conscients "qu'il y a des liens entre la gestion des hommes et la compétitivité ou les résultats économiques de l'organisation" (Louart 1990a: 3), les dirigeants oublient que l'introduction de nouvelles manières de faire en gestion des ressources humaines implique une nouvelle manière de voir et de vivre l'organisation. Modifier la manière de faire implique un changement de soi-même; changer sa philosophie de gestion entraine une modification de la culture de l'entreprise. 
Il ne sert à rien d'intervenir sur les structures, de chercher à accroître la productivité, de tenir compte du système ouvert dans lequel évolue l'organisation et d'établir une stratégie cohérente en fonction de la culture de l'entreprise et des objectifs à atteindre si l'on considère la ressource humaine au même titre que la ressource physique et qu'on pense pouvoir appliquer à sa guise dans son milieu des modèles théoriques. "L'effervescence intellectuelle qui génère un changement de l'horizon du monde n'est pas suffisante pour modifier la réalité " (Crozier 1989: 43). Serait-ce que la période étudiée n'aurait été qu'une décennie du discours? Il serait trop simple de répondre par l'affirmative car il y a eu effectivement une évolution de la fonction ressources humaines. C'est son rythme qui retient notre attention.

\section{CONCLUSION}

L'intérêt envers l'évolution de la fonction ressources humaines ne sera pas stimulé par le souci philanthropique des dirigeants d'entreprises de donner à leur personnel un meilleur milieu de travail ; au contraire, c'est sous la contrainte qu'ils réagiront aux impératifs du moment en les obligeant à se préoccuper davantage des ressources humaines s'ils veulent atteindre leurs objectifs organisationnels.

Les directeurs généraux qui, pour la plupart, ont introduit dans leur discours cette préoccupation à l'endroit des ressources humaines, doivent remettre en cause le partage de l'autorité dévolue à chaque niveau. S'ils désirent introduire une nouvelle philosophie de gestion, basée sur une participation accrue des employés au devenir de l'entreprise, ils doivent remettre en question le partage du pouvoir, c'est-à-dire un plus grand partage de l'information, une reconnaissance réelle du statut des employés, une communication améliorée par et pour des gens mieux formés.

L'évolution de la fonction ressources humaines passe aussi par des changements majeurs dans la culture organisationnelle. Cette nouvelle culture doit privilégier les ressources humaines dans la gestion du changement. Sinon, toute intervention en ce domaine ne servirait qu'à réglementer des processus et à fixer dans le temps la capacité des dirigeants à s'ajuster aux nouvelles situations créées par une organisation en mouvement mais aussi, par des individus dans une société en bouleversement.

Au-delà du désir et de la volonté de la haute direction de donner une place privilégiée à la fonction ressources humaines dans l'organisation, son évolution dépend aussi de son responsable. Mieux formé, plus professionnel, il doit avoir une vision organisationnelle, c'est-à-dire comprendre 
l'impact sur toute l'entreprise de tout changement planifié impliquant davantage les ressources humaines. En plus de posséder les connaissances dans son domaine d'activités, cet agent de changement interne se doit d'avoir une vision globale de la gestion. Il doit être capable d'inciter les gestionnaires à vivre davantage la réalité organisationnelle avec leurs employés en partageant avec ceux-ci plus d'information et plus de pouvoir.

S'il accepte de ne pas s'isoler dans un service des ressources humaines hiérarchisé et désengagé mais plutôt de s'intégrer au fonctionnement général de l'organisation, ce directeur des ressources humaines pourra jouer un rôle moteur dans le développement de la fonction ressources humaines. Ce n'est pas lui qui disparaîtra, mais le phénomène d'une séparation entre les personnes et ceux qui les gèrent va s'estomper avec le temps.

En adoptant une approche d'ouverture, de liberté et d'initiative face à la gestion des ressources humaines, le directeur des ressources humaines se voit confronter au défi de changer les mentalités tout en respectant les gestionnaires en place dans l'entreprise, le personnel et les objectifs poursuivis par l'organisation. De cet angle, il n'est pas surprenant de constater que, depuis 1978, la fonction ressources humaines a évolué lentement au Québec; ce qui surprend, c'est qu'elle ait évolué.

\section{- BIBLIOGRAPHIE}

CASCIO, Wayne, F. 1986. Managing Human Resources: Productivity, Quality of Work Life, Profits. New York, Montréal: McGraw-Hill, 625 p.

CROZIER, Michel. 1989. L'entreprise à l'écoute : Apprendre le management postindustriel. Paris : InterÉditions, 217 p.

GOSSELIN, Louis. 1979. "La fonction personnel dans l'entreprise québécoise, aspect global et aspects particuliers: les perceptions que les directeurs généraux et les directeurs du personnel s'en font ". Thèse de maitrise. Québec: Faculté des sciences sociales, Université Laval, 179 p.

GOSSELIN, Louis. 1991. "La fonction ressources humaines dans l'entreprise: perceptions et évolution. Le cas du Québec", tomes I et II. Thèse de doctorat en sciences de gestion. Lille (France): Institut d'administration des entreprises, $525 \mathrm{p}$.

HERMEL, Philippe. 1990. "Quel management des ressources humaines pour les années 1990?" Personnel, $\mathrm{n}^{\circ}$ 312, 13-20.

LEBOTERF, Guy. 1988. Le schéma directeur des emplois et des ressources humaines: un outil de management stratégique. Paris: Éditions d'Organisation, $233 \mathrm{p}$.

LEMELIN, Maurice et Alain RONDEAU. 1990. "Les nouvelles stratégies de gestion des ressources humaines". Nouvelles stratégies en gestion des ressources 
humaines, textes colligés par Michel Leclerc. Québec: Presses de l'Université du Québec, 83-105.

LOUART, Pierre. 1990a. "L'homme à gérer dans l'organisation : discours des ressources humaines et complexité de l'être humain ». Lille (France) : Institut d'administration des entreprises (non publié), $35 \mathrm{p}$.

LOUART, Pierre. 1990b. "Trois paradoxes en gestion des ressources humaines ". Lille (France): Institut d'administration des entreprises (non publié), $16 \mathrm{p}$. MAHÉ DE BOISLANDELLE, Henri. 1988. "Améliorer la gestion du personnel dans les P.M.E. "Revue française de gestion, $\mathrm{n}^{\circ}$ 68, 93-105.

MARTIN, Claude. 1990. "Contradictions de la fonction personnel : efficacité et méthodes de travail ". Personnel, $\mathrm{n}^{\circ} 315,20-22$.

Milkovich, George-T, William F. GLuECK, Richard T. BARTH et Steven L. MCSHANE. 1988. Canadian Personnel/Human Resource Management: A Diagnostic Approach. Plano, Texas: Business Publications, 877 p.

MiNTSBERG, Henry. 1984. Le manager au quotidien: les dix rôles du cadre. Paris et Montréal : Éditions d'Organisation et Éditions Agence d'Arc, 220 p. PERETTI, Jean-Marie. 1989. "Profil du DRH aujourd'hui ". Ressources humaines, $n^{\circ} 14,30-31$.

PERETTI, Jean-Marie. 1990. Ressources humaines. Collection "gestion ". Paris : Vuibert, $573 \mathrm{p}$.

SCHWARTZ, Robert H. 1985. "Practitioners' Perception of Factors Associated with Human Resource Planning ". Human Resource Planning, vol. 8, $n^{\circ} 2$, 55-66.

ST-ONGE, Sylvie. 1985. La stratégie organisationnelle et l'importance accordée à la GRH. Montréal: Hautes études commerciales, $244 \mathrm{p}$.

ST-PIERRE, Guy. 1989. "Les leaders du génie-conseil canadien sont bien placés pour une expansion rapide (propos recueillis par Françoy Roberge) ". Revue l'Excellence, vol. 2, n 4, 28-29, 53.

TsuI, Anne S. 1984. "Personnel Department Effectiveness: A Tripartite Approach *. Industrial Relations, vol. 23, $\mathrm{n}^{\circ}$ 2, 184-197.

Tsul, Anne S. 1987. "Defining the Activities and Effectiveness of Human Resource Department : A Multiple Constituency Approach ". Human Resource Management, vol. 26, $\mathrm{n}^{\circ} 1,35-69$

WILS, Thierry et Christiane LABELLE. 1989. Efficacité d'un service de ressources humaines. Document de travail, $\mathbf{n}^{\circ}$ 89-04. École de relations industrielles, Université de Montréal, 19 p.

WILS, Thierry, Christiane LABELLE, Gilles GUÉRIN et Jean-Yves LE LOUARN. 1989. "La gestion stratégique des ressources humaines: un reniement du rôle social de l'entreprise?" Relations industrielles/Industrial Relations, vol. 44, $n^{\circ} 2,354-375$. 


\section{SUMMARY}

\section{Perception and Evolution of the Human Resource Function in Quebec}

During the last decade, researchers have developed new theoretical approaches to human resources. The growing preoccupation with the human resource function in the organization has been praised publicly by practitioners. Over the years, this preoccupation has been responsible for changing behavioral patterns as well as human resource management activities.

In 1978, the author conducted a study investigating the human resource function, based on the perceptions of directors and human resource managers. The present study considers the following question: "Have the human resource function in the workplace and the general and specific perceptions of directors and human resource managers evolved over the last decade?"

Three specific dimensions stand out here: the notion of the evolution of human resource in itself; the evolution in the perceptions of the participants; and the time-lag between theory and practice. From these dimensions emerge three hypotheses which revolve around three elements: participants, time and space. This study focuses on the participants' perceptions of the importance of the HR function and covers a period of ten years, 1978-1989. The study establishes a link between the theoretical evolution of the HR function and its applications.

The results of this study are based on the comments of directors and human resource managers in organizations with 200 or more employees. These organizations are located in the province of Quebec and belong to the private secondary sector. The participants completed a questionnaire on general variables, such as the period of time devoted to human resources activities, the influence-authority of the parties involved, interdepartmental communication, the participants' involvement in organizational activities, the right to information and consultation. Questions were also asked on specific variables such as planning of personnel, recruitment in human resources, training and development of human resources.

In our 1978 study, as in that of 1989, information was collected from workplaces representative of the population studied. The profiles of both directors and human resource managers have developed at the same rate as the organizations which employ them. In the 1989 study, however, participants are slightly younger and the academic level of the human resource manager is superior to that of his predecessors. 
Statistical analysis of the collected data allows us to assert that the human resource function in Quebec, including both general and specific characteristics, has progressed very slowly during the last decade. If both structure and activities of this function have increased, it has not, for all that, become more integrated in the evolution of the organization. The analysis also shows that the perception of all participants is more or less the same in 1989 as it was in 1978.

Moreover, integration of the human resource function theoretical concepts did not follow the growth rate expected by both researchers and administrators. Does this mean that, in the last decade, actual developments in human resources lagged behind the level of discourse? An affirmative answer to this question would be too simple since there has nonetheless been some evolution of the human resource function. We are more concerned with the rate of its evolution. In conclusion, the author is considering the possible causes of this reality, and is looking for means to accelerate the evolutionary processes of this function.

$50^{\mathrm{e}}$ congrès des relations industrielles de l'Université Laval

LA RÉORGANISATION DU TRAVAIL EFFICACITÉ ET IMPLICATION

\section{Hôtel Loews Le Concorde, Québec $1^{\text {er }}$ et 2 mai 1995}

Cercles de qualité, ISO 90001-2-3, qualité totale, réingénierie et... quoi encore? Le Québec en chantier

La réorganisation du travail d'un secteur à l'autre

L'individu et la réorganisation du travail

L'organisation du travail au quotidien

Impacts sur l'organisation

De la réorganisation du travail au partenariat régional

Conférence de la ministre de l'Emploi et de la Concertation

Atelier post-congrès sur la réorganisation du travail, 3 mai 1995

Comité d'organisation : Rodrigue Blouin, René Boulard, Paul-André Lapointe, Alain Larocque, Jacques Mercier et Sylvie Montreuil

Information: Congrès des relations industrielles, Faculté des sciences sociales Pavillon Charles-De Koninck

Université Laval, Québec, Canada G1K 7P4 tél. (418) 656-2832/3544 téléc. (418) 656-3316 